\title{
Erratum
}

\section{Erratum to: How Study Design Influences the Ranking of Medicinal Plant Importance: A Case Study from Ghana, West Africa}

\author{
Katherine Dudney ${ }^{*}, 1$, Sarah Warren ${ }^{2}$, Erin Sills $^{2}$, and Jerry Jacka ${ }^{3}$
}

${ }^{1}$ AECOM, 1333 Broadway, Suite 800, Oakland, CA 94612, USA

${ }^{2}$ Department of Forestry and Environmental Resources, North Carolina State University, 2800 Faucette Drive, Campus Box 8008, Raleigh, NC 27695, USA

${ }^{3}$ Department of Anthropology, University of Colorado, Boulder, 1350 Pleasant St., Campus Box 233

UCB, Boulder, CO 80309-0233, USA

*Corresponding author; e-mail: katie.lists@gmail.com

\section{Erratum to: Economic Botany DOI 10.1007/s12231-015-9322-y}

The version of this article initially published contained the following errors. Table 6 (p. 314) was incorrectly inserted as a repeat of Table 5 . The correct Table 6 is presented below.

On Figure 1 (p. 309), villages are marked with circles while settlements are marked as squares.
The Cultural Value Index equation (p. 310) should be:

$$
C V_{S}=\left[{ }^{N U_{S} / N} C\right] \times\left[{ }^{R_{S}}{ }_{N}\right] \times\left[\frac{\sum_{u} F C_{u s}}{N}\right]
$$

TABLE 6. CORRELATION MATRIX FOR INDEX RESULTS FOR PROMPTED VS. UNPROMPTED RESPONSES.

\begin{tabular}{|c|c|c|c|c|c|c|c|c|c|c|c|c|c|}
\hline & \multicolumn{6}{|c|}{ Unprompted } & \multicolumn{6}{|c|}{ Prompted } \\
\hline & & Freq & \#Uses & $\mathrm{CVs}$ & RI & IAS & LIC & Freq & \#Uses & $\mathrm{CVs}$ & RI & IAS & LIC \\
\hline \multirow{6}{*}{ 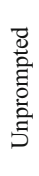 } & Freq & 1.00 & 0.96 & 0.99 & 0.99 & 0.93 & 1.00 & 0.47 & 0.44 & 0.50 & 0.49 & 0.33 & 0.46 \\
\hline & \# Uses & & 1.00 & 0.98 & 0.99 & 0.82 & 0.95 & 0.45 & 0.44 & 0.48 & 0.47 & 0.30 & 0.44 \\
\hline & $\mathrm{CVs}$ & & & 1.00 & 1.00 & 0.89 & 0.99 & 0.47 & 0.46 & 0.50 & 0.49 & 0.33 & 0.46 \\
\hline & RI & & & & 1.00 & 0.89 & 0.98 & 0.47 & 0.45 & 0.50 & 0.49 & 0.32 & 0.46 \\
\hline & IAS & & & & & 1.00 & 0.95 & 0.36 & 0.31 & 0.37 & 0.37 & 0.24 & 0.35 \\
\hline & LIC & & & & & & 1.00 & 0.46 & 0.43 & 0.49 & 0.48 & 0.32 & 0.45 \\
\hline \multirow{6}{*}{ 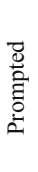 } & Freq & & & & & & & 1.00 & 0.89 & 0.98 & 0.99 & 0.92 & 1.00 \\
\hline & \# Uses & & & & & & & & 1.00 & 0.96 & 0.93 & 0.72 & 0.88 \\
\hline & $\mathrm{CVs}$ & & & & & & & & & 1.00 & 0.99 & 0.86 & 0.97 \\
\hline & RI & & & & & & & & & & 1.00 & 0.88 & 0.99 \\
\hline & IAS & & & & & & & & & & & 1.00 & 0.94 \\
\hline & LIC & & & & & & & & & & & & 1.00 \\
\hline
\end{tabular}

The online version of the original article can be found at http://dx.doi.org/10.1007/s12231-015-9322-y 